\title{
Dual Watermarking Technic using Chaotic Map And Discrete Wavelet Transform
}

\author{
Ramafiarisona Hajasoa Malalatiana \\ Telecommunication-Automatic-Signal-Image-Research \\ Laboratory/Doctoral School in Science and Technology of Engineering and Innovation/University of Antananarivo \\ Antananarivo, Madagascar
}

\begin{abstract}
There is an increasing need of security for information and communication due to the growth of internet users. Many techniques are available to satisfy these needs such as cryptography, steganography or watermarking. We propose a dual scheme for image watermarking by combining encryption and multi-resolution analysis. This mechanism has advantages of providing both high security and low time complexity. It uses chaotic encryption with logistic map, alpha fusion method and discrete wavelet transform (DWT) algorithm both together. The main objective is to achieve high security, high Peak Signal to Noise Ratio (PSNR) value, good imperceptibility and robustness.
\end{abstract}

Keywords-Watermarking; Encryption, Logistic map; Alpha fusion; DWT(Discrete Wavelet Transform).

\section{INTRODUCTION}

Nowadays, Information security and confidentiality become unavoidable problems and cannot be ignored.

This paper uses a new scheme based on two techniques: cryptography and watermarking.

Watermarking techniques are appropriate for copyright protection. It embeds an invisible signature into a host source. Some requirements are needed like imperceptibility of the hidden signature, robustness against data processing and attack, capacity of hiding. In this case, the embedding method is based on wavelet transform and alpha fusion.

\section{CHAOTIC ENCRYPTION AND DECRYPTION}

\section{A. Principle}

Chaotic systems are particular class of non linear system. A chaotic system must obey some conditions [1][2]:

- Non linearity

- Determinism

- Sensibility of initial values

We obtain a chaotic function by iteration of simple mathematical function. Many functions can be used like logistic recurrence (1), sine recurrence (2), standard recurrence (3), etc [3][4].

$$
\begin{aligned}
& \mathrm{X}_{\mathrm{n}+1}=\text { alpha } * \mathrm{X}_{\mathrm{n}}\left(1-\mathrm{X}_{\mathrm{n}}\right) \\
& \mathrm{X}_{\mathrm{n}+1}=\text { beta } * \sin \left(\mathrm{pi} * \mathrm{X}_{\mathrm{n}}\right) \\
& \left\{X_{n+1}=X_{n}+\text { gamma } * \sin Y_{n}\right. \\
& \mathrm{Y}_{\mathrm{n}+1}=\mathrm{Y}_{\mathrm{n}}+\mathrm{X}_{\mathrm{n}+1}
\end{aligned}
$$

Encryption and decryption of the signature is performed by a two-dimensional logistic map that Eq (4) defines [4][5][6]:

$$
\left\{\begin{array}{l}
X_{n+1}=r *\left(3 * Y_{n}+1\right) * X_{n} *\left(1-X_{n}\right) \\
Y_{n+1}=r *\left(3 * X_{n}+1\right) * Y_{n} *\left(1-Y_{n}\right)
\end{array}\right.
$$

$\mathrm{X}$ and $\mathrm{Y}$ contain elements as much as signature length: After this step, we rearrange $\mathrm{X}$ and $\mathrm{Y}$ to match the size of the signature. Results are used for diffusion and confusion.

\section{B. Diffusion method}

Maps $\mathrm{X}$ and $\mathrm{Y}$ are summed and converted to decimal numbers by an approximation, we obtain the map S. A function is preformed to each sub block of 4 by 4 of $\mathrm{S}$, the result is noted $\mathrm{T}$. Finally, we sum the mark $\mathrm{M}$ with $\mathrm{T}$ to get the cipher signature with diffusion method. It can be illustrated:

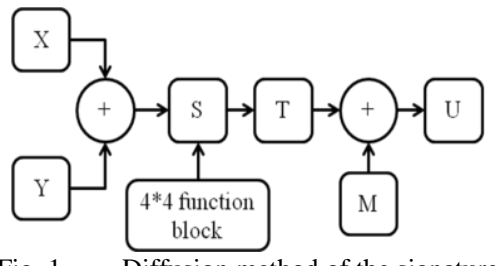

Figure 2 shows how the diffusion method using this twodimensional logistic map affects the mark and its histogram.
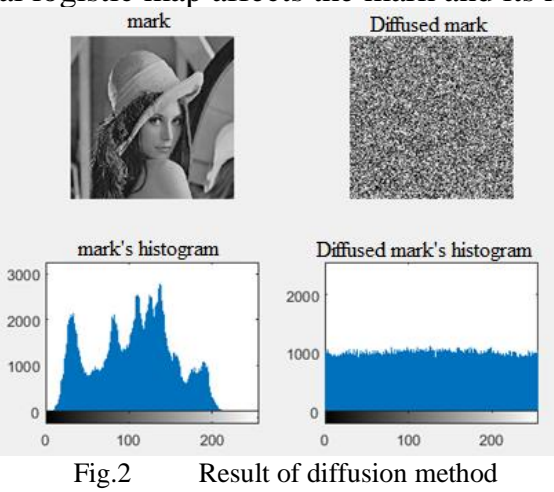

Statistically, the histogram of the cipher image is uniform. The mark is substituted by a chaotic generator with couple of initials values (xo, yo).

The mark is perfectly recovered when we strictly use the same couple of initials values on the restitution process.

\section{Confusion method}

$\mathrm{X}$ is used to mix pixel in the same row, and $\mathrm{Y}$ to the same column. First, the map $\mathrm{X}$ is sorted on its row to get a matrix containing the indexation of this sort: $\mathrm{Xp}$. Then, the mark is permuted row by row following the layout of $\mathrm{Xp}$ : we obtain $\mathrm{V}$. We process in the same way for column by $\mathrm{Y}$. This method is illustrated on Fig.3. 


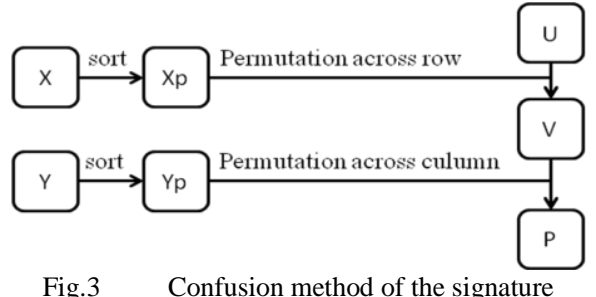

Figure 4 shows the permutation process of $M$ instead of $U$ so we can visualize how it works.

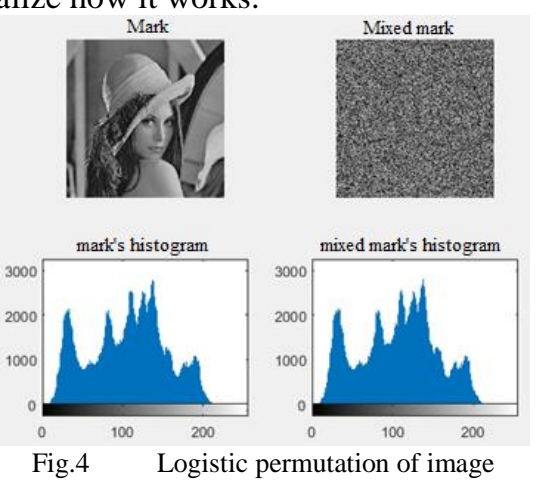

\section{DISCRETE WAVELET TRANSFORM}

In order to combine strengths of spatial and frequency domain watermarking, we use a multi-resolution analysis which provides a spatial-frequency representation of image. The wavelet transform is of this type.

\section{A. Continuous Wavelet Transform}

The Continuous Wavelet Transform (CWT) uses the same principle as Short Term Fourier Transform analysis. However, there are two main differences between them [8][9]:

- Analyzer function isn't the same: for STFT, the analyzer is Fourier function; for CWT, the analyzer function is a wavelet.

- The width of the window is changed as the transform is computed.

1) Wavelet

The base function of CWT is the wavelet. It's a function that graphically represents a little wave. We get the transformation by convolution of a contracted, dilated, and translated wavelet.

A function $\psi$ is a wavelet if

$$
C_{\psi}=\int_{-\infty}^{+\infty} \frac{|\hat{\psi}(f)|^{2}}{|f|} d f<\infty
$$

Where $\psi$ (f) is the FT of $\psi(\mathrm{t})$. It is the admissibility condition.

2) $C W T$

Analyzer functions are defined by dilatation and translation of the main wavelet.

$$
\psi_{a b}(t)=\psi\left(\frac{t-b}{a}\right), a \neq 0
$$

$\mathrm{b}$ is the translation parameter

$a$ is the scale factor

So, CWT of signal $\mathrm{f}(\mathrm{t})$ is:

$$
F(a, b)=\frac{1}{\sqrt{a}} \int_{-\infty}^{+\infty} f(t) \overline{\psi_{a, b}}(t) d t
$$

Reconstitution of $\mathrm{f}(\mathrm{t})$ is given by:

$$
f(t)=\frac{1}{c_{\psi}} \int_{-\infty}^{+\infty} \int_{-\infty}^{+\infty} \frac{f(a, b)}{a^{2}} \psi_{a, b}(t) d a d b
$$

\section{B. Discrete Wavelet Transform}

\section{1) $1 D \mathrm{DWT}$}

Discrete Wavelet Transform (DWT) uses filtering to analyze signals. An analyze process is based on dividing the signal in to 2 sub band: a half for low frequency and another half for high frequency. A decimation by 2 is used after filtering. We can compute many processing as needed by taking the low frequency of the previous process as new parameter. Fig.5 shows how an analysis works. [9][10][11].

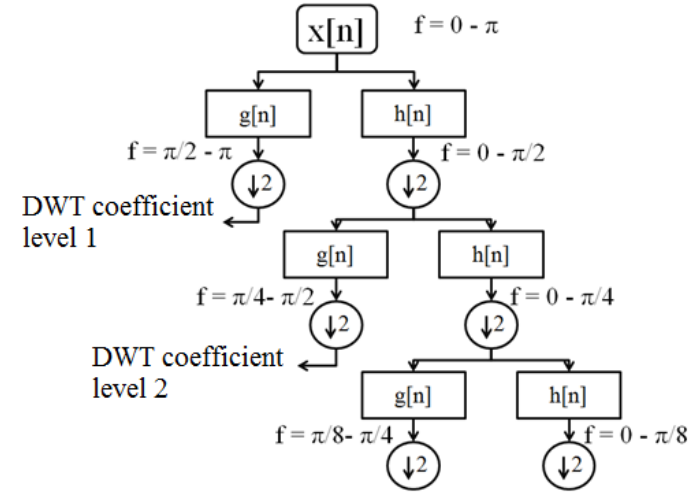

Fig.5 Analysis with DWT

\section{2) $2 D D W T$}

For two dimensional signals, each decomposition level contains two levels:

- Analysis across row vector which give 2 signals. The decimation by 2 permits to reduce information by half so results are the same size as original.

- Analysis across column vector.

A decomposition level gives one matrix of approximation LLX (stands for Low Low level X) and three matrixes of details: LHX, HLX, HHX (respectively Low High level X, High Low level X, High High level X).[10][11][12]

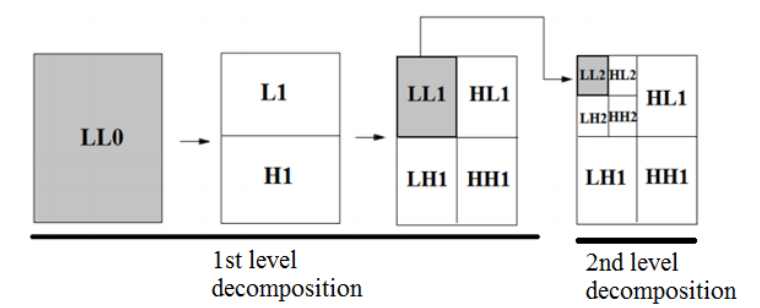

Fig.6 Representation of approximations and details coefficients

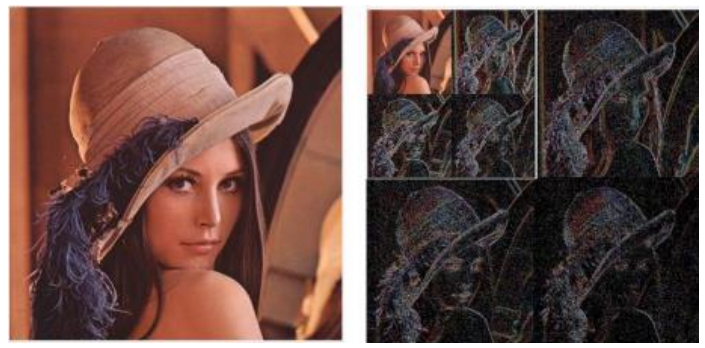

Fig.7 Wavelet decomposition of an image

IV. PROPOSED WORK

An algorithm of watermarking using chaotic map and DWT decomposition is discussed in this section. Its 
performance is quantified by insertion capacity, robustness against attack and imperceptibility.

\section{A. Performance measurements \\ 1) Capacity}

The capacity is the ratio between the volume of signature and the size of cover image.

$$
C=\frac{M(\text { bit })}{I_{\text {row }} * I_{\text {col }} \text { (pixel) }}(\text { bpp })
$$

$\mathrm{M}$ is the size of the signature expressed in Bit.

The denominator is the size of the host image expressed in number of pixel.

\section{2) Imperceptibility}

Imperceptibility is quantified by Mean Square Error (MSE) and Peak Signal to Noise Ratio (PSNR)

$$
\begin{gathered}
P S N R=10 \log _{10} \frac{255^{2}}{M S E} \\
M S E=\left(\frac{1}{M * N}\right) \sum_{i=1}^{M} \sum_{j=1}^{N}\left(a_{i j}-b_{i j}\right)^{2}
\end{gathered}
$$

$\mathrm{M}$ and $\mathrm{N}$ denote row and column size of image $\mathrm{a}$ and $\mathrm{b}$.

Higher the PSNR, better the imperceptibility is.

\section{3) Robustness}

Mathematically, robustness is expressed by the correlation between the original mark and the extracted mark from watermarked image.

$$
r=\frac{\sum_{i} \sum_{j}\left(A_{i j}-\bar{A}\right)\left(B_{i j}-\bar{B}\right)}{\sqrt{\left(\sum_{i} \sum_{j}\left(A_{i j}-\bar{A}\right)^{2}\right)\left(\sum_{i} \sum_{j}\left(B_{i j}-\bar{B}\right)^{2}\right)}}
$$

Where $\mathrm{A}$ and $\mathrm{B}$ are images to be compared.

\section{B. Embedding process}

The signature is encrypted using logistic map. The cover image is decomposed in 2 levels by DWT. We embed the cipher mark in a level 2 sub band by alpha fusion method.

Alpha fusion method is a weighted sum of two matrix described as follow:

$$
\begin{gathered}
X^{\prime}=\alpha X+\beta W \\
\alpha+\beta=1 \text { and } \alpha \gg \beta
\end{gathered}
$$

$\mathrm{X}$ is the level 2 DWT decomposed host image.

$\mathrm{W}$ is the encrypted signature.

Alpha is the integration coefficient and represents the strength of the watermark. Fig. 8 shows the diagram of embedding process.

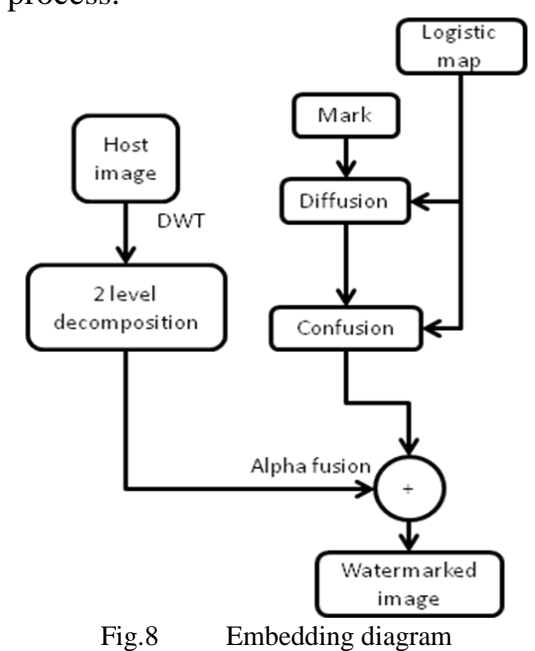

Fig.8 Embedding diagram

\section{Results and Discussions}

This section is dedicated on performance measurement. Host source is the image "lena.tif" and the mark is a grayscale image.

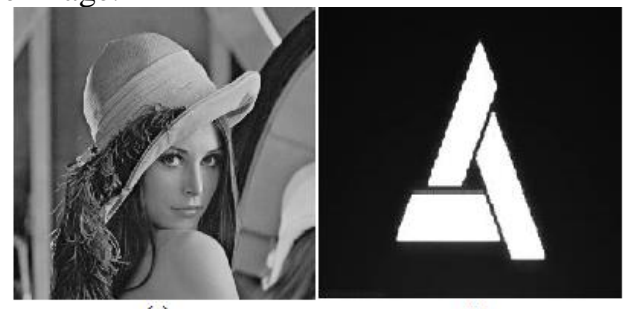

(a)

(b)

Fig.9 (a) host image, (b) mark

The capacity of insertion is equal to the size of the support sub band. In level 2, the capacity is the quarter of host image.

1) Influence of insertion sub band and alpha

Imperceptibility depends on two factors: the insertion sub band and the integration coefficient. Fig.13 shows their relation.

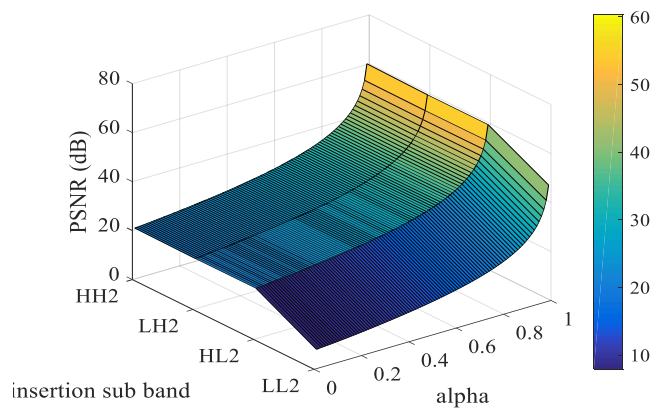

Fig.10 PSNR depending on integration sub band and alpha

LL2 sub band contains the lowest frequencies of the image. So an insertion on this sub band drives to many bad effects on imperceptibility.

PSNR of the image increases with alpha. Human sight doesn't see differences between the original image and the watermarked if PSNR is above $40 \mathrm{~dB}$.

2) Robustness measurements

This part tests the robustness of watermark against erasure and geometrical attack.

The watermarked image will be tested by:

- Erasure attack

- Geometrical attack

a) Results against noise

This section evaluates the robustness of the watermark when we add noise. Gaussian noise is used.

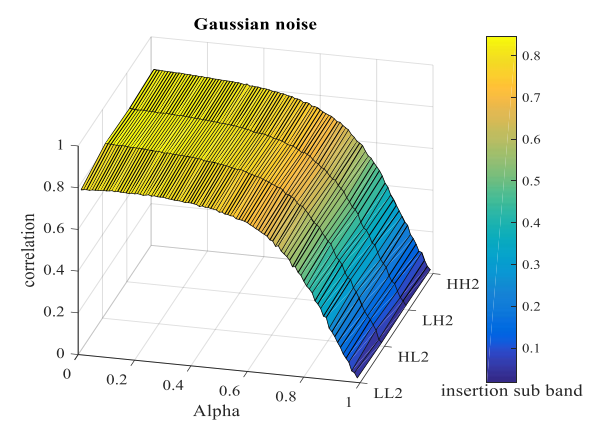

Fig.11 Robustness against Gaussian noise 
The mark is robust against noise for little values of alpha. $\mathrm{HH} 2$ is the best insertion support.

b) Results against filtering

Median filter is used for tests.

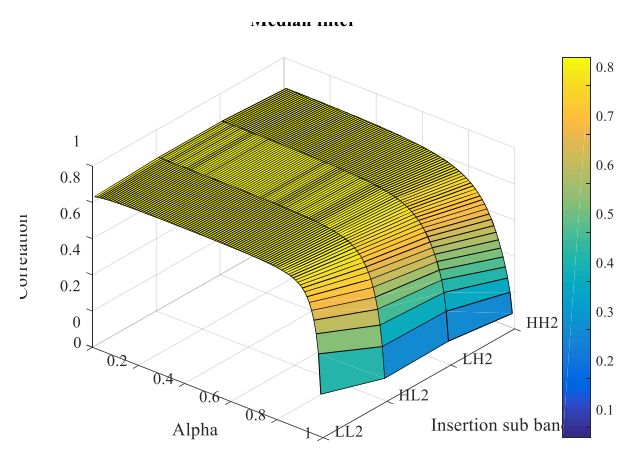

Fig.12 Robustness against median filter

The algorithm is especially robust against filtering. Correlations remain constant for alpha values below 0.8 . They suddenly decrease above this value but the mark is still recognizable.

c) Results against rotation

Figure 13 shows correlation results.
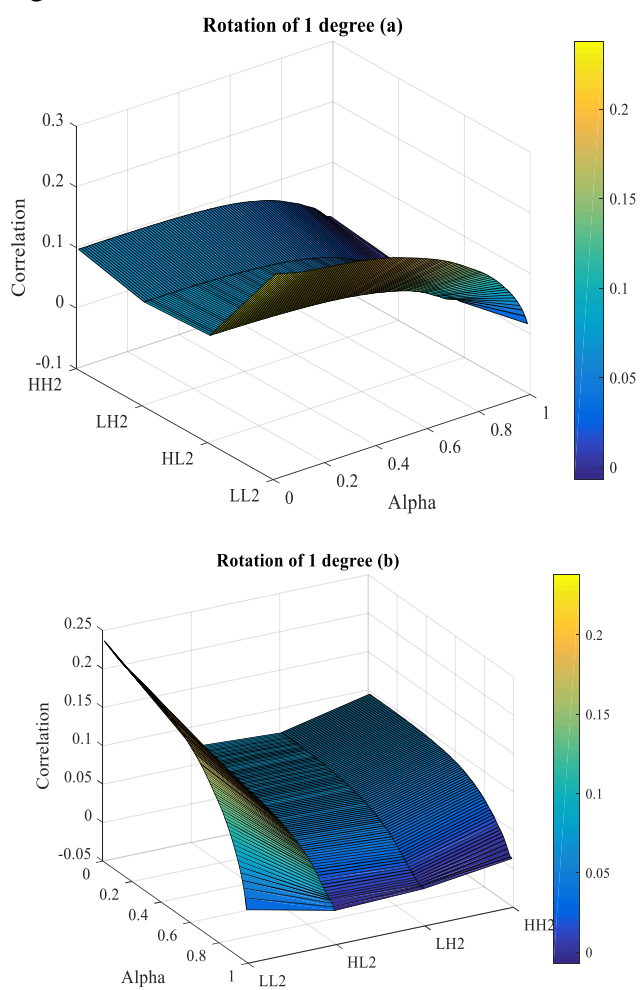

Fig.13 Robustness against rotation

Correlations don't exceed 0.25 . It isn't enough to recover the signature. A slightest rotation drives to an undetectable and unrecognizable mark.

\section{CONCLUSION}

Applied to signatures, encryption using chaotic system with logistic map is very fast, easy to compute and provides high level of security.

Restriction of old embedding process led us to apply a multiresolution analysis tool for watermarking, especially DWT. It requires the original image to extract the mark. DWT based watermarking scheme appears to be resistant on erasure attack.

\section{REFERENCES}

[1] D E Goumidi, «Fonction logistique et standard chaotique pour le chiffrement des images satellitaires », Diploma thesis, Mentouri University, 2010

[2] S J Clinton, "Chaos and time-series analysis", Oxford Universities Press, 2003

[3] F Ansett, "Les systems dynamiques chaotiques pour le chiffrement: synthèse et cryptanalyse", Nancy's Automatic Research Center, 2005

[4] J Friedrich, "Symmetric ciphers based on two-dimensional chaotic maps", Chaos Solitons and Fractals, 1998

[5] J Gleick, "La théorie du chaos vers une nouvelle science", Champs Flammarion, 1991

[6] J Friedrich, "Image encryption based on chaotic maps", IEEE Conference on System, Man and Cybernetics, 1997

[7] M Antonini, Daubechies, "Image coding using wavelet transform"

[8] R Polikar, "fundamental concept \& overview of the wavelet theory", Dept. of Electrical and Computer Engineering, 1996

[9] I Daubechies, "Ten lectures on wavelets", Regional Conference Series in Applied Mathematics, Philadelphia, 1992

[10] R Alt, "La transformation en ondelettes", Pierre and Marie Curie University, 2001

[11] C Rey and J L Dugelay, "An overview of watermarking algorithms for image authentification”, EUROCOM Institute, 2000

[12] P Y Chen and H J Lin, "A DWT based approach for image Steganography", International Journal of Applied Science and Engineering, 2006 